\title{
X-ray Pulsars in the Small Magellanic Cloud
}

\author{
R. H. D. Corbet ${ }^{1,2}$, M. J. Coe ${ }^{3}$, J. Galache ${ }^{3}$ S. Laycock ${ }^{4}$, \\ C. B. Markwardt ${ }^{1,5}$ and F. E. Marshall ${ }^{1}$ \\ ${ }^{1}$ X-ray Astrophysics Laboratory, Code 662, \\ NASA/Goddard Space Flight Center, Greenbelt, MD 20771, USA \\ ${ }^{2}$ Universities Space Research Association \\ ${ }^{3}$ Department of Astronomy, University of Southampton, \\ Southampton, SO17 1BJ, UK \\ ${ }^{4}$ Center for Astrophysics, 60 Garden Street, Cambridge, MA 02138, USA \\ ${ }^{5}$ University of Maryland
}

\begin{abstract}
The SMC is now known to contain many more transient X-ray pulsars than would be expected based on a simple scaling of the number of such sources in the Galaxy by the relative mass of the SMC. We have been conducting regular monitoring observations of the SMC with the Proportional Counter Array on the Rossi X-ray Timing Explorer since 1997. This has resulted in the discovery of many of these X-ray pulsars and also provided orbital period measurements from detections of regular outbursts. We can now investigate the differences and similarities of the Galactic and SMC X-ray pulsar populations and consider the origin of the huge SMC X-ray pulsar over-abundance.
\end{abstract}

Keywords. galaxies: Magellanic Clouds, X-rays: binaries, stars: neutron.

\section{Introduction}

The first known X-ray pulsar in the SMC was the persistent supergiant system SMC X-1. Two luminous transients (SMC X-2, SMC X-3) were discovered with SAS-3 (Clark et al. (1978)). These were thought to be transient Be/neutron star systems although pulsations were not detected due to the low sensitivity of SAS-3. It was hypothesized that SMC pulsars were exceptionally luminous, possibly related to the low metallicity of the SMC (Westerlund (1990)). This was later to be disproved and an alternative explanation found for the high luminosity of the first few SMC X-ray pulsars to be discovered. Over subsequent years a few pulsars were also found with satellites such as ROSAT (Hughes (1994)).

\section{SMC X-ray pulsars with RXTE}

Serendipitous RXTE slew observations in 1997 showed a possible outburst from the vicinity of SMC X-3. Follow up target of opportunity pointed RXTE observation showed a complicated power spectrum with several peaks that were not all harmonically related to each other. Imaging ASCA observations were next made which showed two separate pulsars, however neither was found to be located at the position of SMC X-3. A more detailed look at the RXTE power spectrum showed that in fact three pulsars were simultaneously active (Corbet et al. (1998)). These observations were the first sign of the existence of a very large SMC X-ray pulsar population. 


\section{The RXTE monitoring program}

RXTE has been regularly monitoring the SMC since 1997 using the Proportional Counter Array (PCA). We have discovered very many transient X-ray pulsars. For those sources where optical counterparts have been identified they are all found to be Be stars. We primarily make weekly observations of one particularly active region near SMC X3. Other SMC regions have been monitored monthly depending on the amount of time awarded in a particular observing cycle. We use power spectra to extract pulsed flux from any pulsars. In this way, although the PCA is not an imaging instrument, the pulsed flux from multiple sources can be monitored independently. When new sources are identified we use cross scans in Right Ascension and Declination to localize the position of the new sources. For new sources we thus obtain at least minimal positional information. However, position determination can be problematic if too many pulsars are simultaneously active. A log of known X-ray pulsars in the SMC is currently maintained at http://lheawww.gsfc.nasa.gov/ corbet/pulsars/. About 45 sources are currently known.

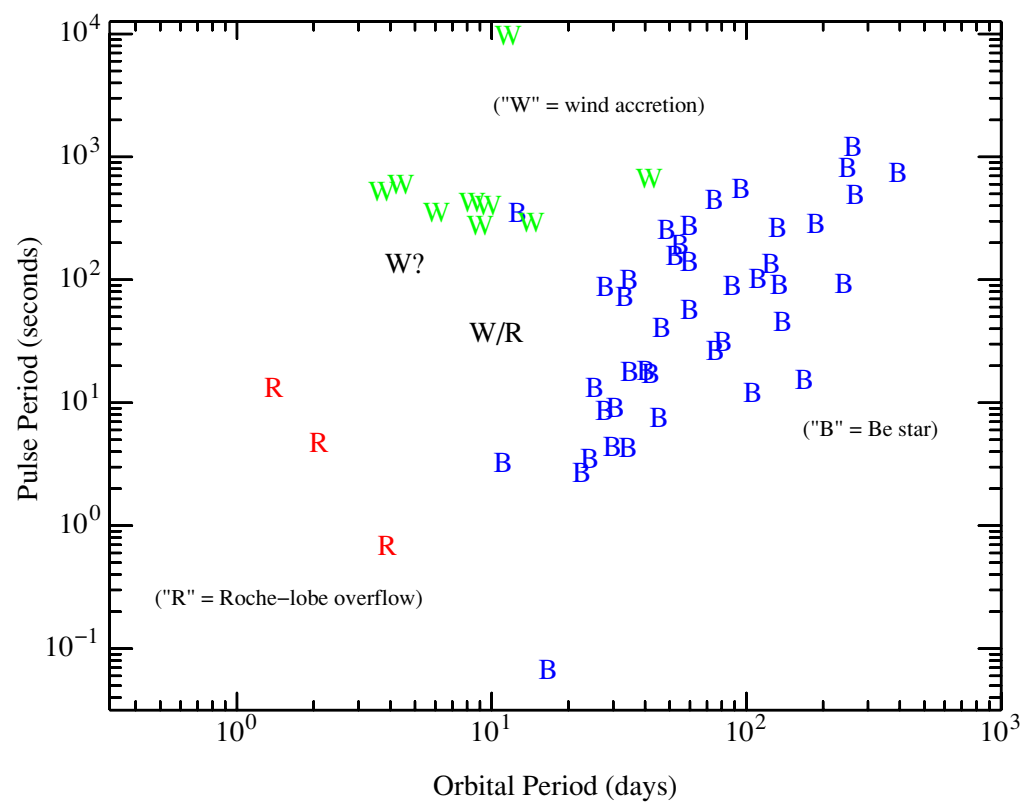

Figure 1. Pulse and orbital periods of HMXB pulsars. This plot includes systems in the Milky Way, SMC, and LMC.

\section{Measuring Orbital Periods}

From our RXTE monitoring program we have been able to determine the orbital period of a number of systems from the detection of regular outbursts. These period measurements are augmented by optical period determinations using OGLE and MACHO light curves (e.g. Schmidtke \& Cowley (2005), Edge et al. (2005)). 


\section{The Be/Neutron Star Spin/Orbital Period Relationship}

When Be HMXBs are in an active state (i.e. the Be star is surrounded by a sufficiently extensive disk) they often show regular X-ray outbursts which reveal the orbital period of the system. In addition, the X-ray light curves also show pulsations on the rotation period of the neutron star. It was first noted over twenty years ago that, for these systems, there is a correlation between pulse period and orbital period (Corbet (1984)). This correlation has been interpreted as arising from the spin period of the neutron star being determined by a balance of spin-up and spin-down torques. The spin period is forced to remain close to a time-averaged value where the corotation and magnetospheric radii are equal (Corbet (1984), Corbet (1986), Waters \& van Kerkwijk (1989)). In this way the spin/orbital period relationship probes the circumstellar envelope around Be stars.

In order to characterize the $\mathrm{P}_{s}$ vs. $\mathrm{P}_{\text {orb }}$ relationship a simple linear fit was made to the $\log$ of these values. As the spin period is believed to be determined by the average local circumstellar environment, which depends on the size of the orbit, the orbital period may be regarded as the independent variable.

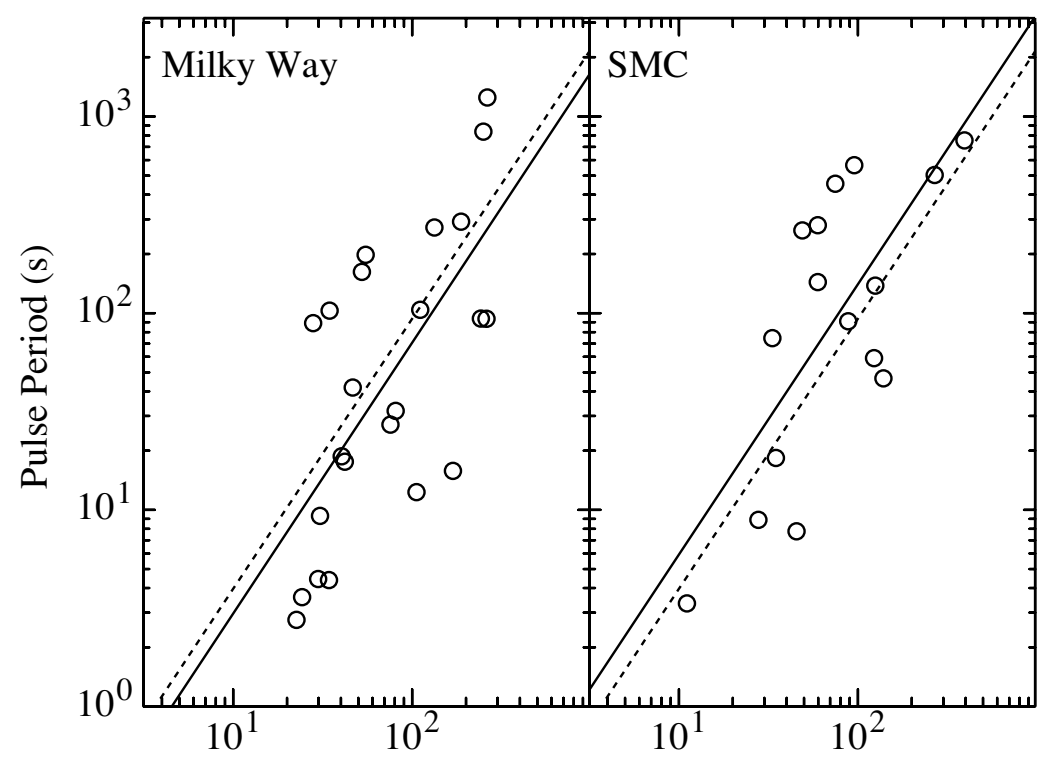

Orbital Period (d)

Figure 2. Pulse and orbital periods of Be/neutron star binaries in the Milky Way and SMC plotted separately. In each panel the dashed lines show the fit to the combined Milky Way/SMC data set and the solid lines show the fits to just the indicated subset.

\section{Discussion}

The relationship between pulse and orbital periods is found to be identical, within the errors, for both Galactic and SMC Be/neutron star binaries. This was not completely expected before hand as there is a number of differences between Galactic and SMC Be stars: (i) Maeder et al. (1999) find that the Be star phenomenon is more common in the SMC, (ii) the metallicity of the SMC is significantly lower than in the Galaxy 
with an overall metallicity of $1 / 5$ solar, and even young clusters have low metallicities (e.g. Hill \& Spite (1999)), (iii) Coe et al. (2005) find from optical photometry that the spectral types of primaries in Be/neutron star systems in the SMC may span a larger range than Galactic systems where the primaries are B2 or earlier. Because of these numerous differences it is somewhat surprising that we see no difference in the overall spin/orbital relationship. For early type supergiant stars where mass loss in winds depends on absorption of UV lines by metals there does appear to be a difference between the SMC and the Milky Way. Theoretically, radiatively driven mass loss is expected to depend on metallicity (Kudritzki et al. (1987), Vink et al. (2001)) as:

$$
\dot{M} \propto Z^{0.5 \rightarrow 0.7}
$$

The Be star mass loss mechanism appears not to be strongly dependent on metallicity and hence, by implication, is not strongly affected by radiation pressure.

\section{References}

Clark, G., Doxsey, R., Li, F., Jernigan, G., \& van Paradijs, J. 1978, ApJ 221, L37

Coe, M., Edge, W., Galache, J., \& McBride, V. 2005, MNRAS, 356, 502

Corbet, R. 1984, A\&A, 141, 91

Corbet, R. 1986, MNRAS, 220, 1047

Corbet, R., Marshall, F., Lochner, J., Ozaki, M., \& Ueda, Y. 1998, IAUC, 220

Edge, W., Coe, M., Galache, J., McBride, V., Corbet, R., Okazaki, A., Laycock, S., Markwardt, C., Marshall, F., \& Udalski, A. 2005, MNRAS, 361, 743

Hill, V. \& Spite, M. 1999, Ap \& Space Sc., 265, 469

Hughes, J. 1994, ApJ, 427, L25

Kudritzki, R., Pauldrach, A., \& Puls, J. 1987, A\&Ap, 173, 293

Laycock, S., Corbet, R., Coe, M., Marshall, F., Markwardt, C., \& Lochner, J. 2005, ApJ in press

Maeder, A., Grebel, E., \& Mermilliod, J.-C. 1999, ApJ, 346, 459

Schmidtke, P.C. \& Cowley, A.P. 2005, AJ, 130, 2220

Vink, J., de Koter, A., \& Lamers, H. 2001, A\& $\mathcal{E} A$, 369, 574

Westerlund, B. 1990, A\&ARv, 2, 29

Waters, L. \& van Kerkwijk, M. 1989, $A \mathscr{E} A p, 223,196$

\section{Discussion}

VAN DEN Heuvel: Could it not be that we are missing many Be/X-ray systems in our Galaxy? They tend to be off for long periods, so we observe only a fraction of them. An over-abundance by a factor 50 for the SMC seems very extreme to me. I could imagine an over-abundance with respect to the Milky Way by a factor a few (perhaps as much as 5) due to a starburst, but not 50 .

CoRBET: Unless systems in the SMC are active for a larger fraction of time than Galactic systems then the effects of sources being off should affect estimates of source populations in both the SMC \& Milky Way in a similar way. Searches for new Be sources in the Milky Way with both the RXTE ASM \& Galactic plane scans with the PCA have not revealed very large numbers of new Be star sources. (However, absorption in the plane of the Galaxy does of course affect the sensitivity of these searches.) We also observed a single position in the LMC with the PCA, already known to contain a few pulsars, once per month for one year. No new pulsars were found, indicating a substantial difference between the LMC \& SMC.

DiStefano: Is it possible that we are missing X-ray pulsars in the Galaxy? 
CoRbet: Please see my reply to the similar question from Van den Heuvel.

ZEZAS: Just a comment: Studies of the star-formation history of the SMC show that there was a recent burst of star-formation $\sim 10-30 \mathrm{Myr}$ ago, which may explain the observed excess of Be-X-ray binary pulsars.

CoRBeT: I agree. Such a recent burst of star formation is reported by Harris \& Zaritsky (2004). Star Formation at that time might explain the surplus of Be star X-ray binaries but lack of excess of the shorter-lived supergiant wind accretors.

GHosh: The discrepancy in the OB HMXB/Be-star binary ratio between SMC \& Milky Way is most interesting and needs to be understood. If we believe that the massive companions from the same generation are in both types, somehow the parts of the massrange or $\mathrm{P}_{\text {orb }}$ range relevant for $\mathrm{OB}$ companions are absent in SMC.

Also a comment: If the $\mathrm{P}_{s}-\mathrm{P}_{\text {orb }}$ correlation is dominated by Be-star binaries, it is not surprising that SMC \& Milky Way are very similar in this respect.

CoRBeT: In looking at the $\mathrm{P}_{s}-\mathrm{P}_{\text {orb }}$ correlation, I only considered sources known to be Be star sources, or strongly suspected to be so because they are transient. The SMC metallicity is a factor $\sim 5$ lower than the Galaxy. If the Be star phenomenon, e.g. mass loss rate or structure of the Be star envelope, depends on metallicity that would affect the correlation. 